\title{
Channel Estimation Performance Analysis of FBMC/OQAM Systems with Bayesian Approach for 5G-Enabled IoT Applications
}

\author{
Han Wang $\mathbb{D}^{1,2}$ Wencai Du $\mathbb{D}^{1},{ }^{2}$ Xianpeng Wang, ${ }^{3}$ Guicai $Y u,{ }^{1}$ and Lingwei Xu $\mathbb{D}^{4}$ \\ ${ }^{1}$ College of Physical Science and Engineering, Yichun University, 576 Xuefu Road, Yichun, Jiangxi 336000, China \\ ${ }^{2}$ Institute of Data Science, City University of Macau, Avenida Padretomas Pereira, Taipa, Macau 999078, China \\ ${ }^{3}$ State Key Laboratory of Marine Resource Utilization in South China Sea, Hainan University, 58 Renming Road, Haikou, \\ Hainan 570228, China \\ ${ }^{4}$ Department of Information Science and Technology, Qingdao University of Science and Technology, 99 Songling Road, Qingdao, \\ Shandong 266061, China
}

Correspondence should be addressed to Han Wang; hanwang1214@gmail.com and Wencai Du; georgedu@city.mo

Received 17 February 2020; Revised 4 May 2020; Accepted 16 May 2020; Published 4 June 2020

Academic Editor: Xingwang Li

Copyright (c) 2020 Han Wang et al. This is an open access article distributed under the Creative Commons Attribution License, which permits unrestricted use, distribution, and reproduction in any medium, provided the original work is properly cited.

\begin{abstract}
A filter bank multicarrier (FBMC) with offset quadrature amplitude modulation (OQAM) (FBMC/OQAM) is considered to be one of the physical layer technologies in future communication systems, and it is also a wireless transmission technology that supports the applications of Internet of Things (IoT). However, efficient channel parameter estimation is one of the difficulties in realization of highly available FBMC systems. In this paper, the Bayesian compressive sensing (BCS) channel estimation approach for FBMC/OQAM systems is investigated and the performance in a multiple-input multiple-output (MIMO) scenario is also analyzed. An iterative fast Bayesian matching pursuit algorithm is proposed for high channel estimation. Bayesian channel estimation is first presented by exploring the prior statistical information of a sparse channel model. It is indicated that the BCS channel estimation scheme can effectively estimate the channel impulse response. Then, a modified FBMP algorithm is proposed by optimizing the iterative termination conditions. The simulation results indicate that the proposed method provides better mean square error (MSE) and bit error rate (BER) performance than conventional compressive sensing methods.
\end{abstract}

\section{Introduction}

To date, the application of mobile communication systems in the field of Internet of Things (IoT) is not in-depth [1,2]. Although Fourth Generation (4G) has greatly improved the network speed, there is still much room for improvement in network reliability and latency. Fifth Generation (5G) systems [3-8] are now being deployed which can well meet the demands of IoT, such as low latency, high reliability network, and large bandwidth. The full opening of the $5 \mathrm{G}$ era is accelerating the application and popularization of IoT, artificial intelligence (AI), and other technologies [9-12]. However, the development of IoT requires suitable infrastructure $[13,14]$ including sensors for data acquisition and wireless communication technology. Currently, multicarrier modulation has been widely used in wireless communication systems. The filter bank multicarrier (FBMC) with offset quadrature amplitude modulation (OQAM), denoted as FBMC/OQAM, has captured significant attention $[15,16]$, due to its potential as an option to orthogonal frequency division multiplexing (OFDM). Although the Third Generation Partnership Project (3GPP) has indicated that filtered OFDM will be utilized in 5G systems, interest in FBMC for future mobile communication systems has not declined $[17,18]$.

FBMC technology employs a good time-frequency prototype filter, which has many features such as low spectral side lobes, high spectrum efficiency, and robustness to frequency offset [19]. However, the nonstrict orthogonality of the system leads to the existence of imaginary interference. This interference can be mitigated during channel estimation (CE), but this requires that the channel coefficients be estimated in the complex domain. Interference reduction is challenging in FBMC systems, particularly when multiple-input multiple-output (MIMO) communications are involved. 
There has been significant effort to overcome this problem, and both preamble-based [20-27] and compressive sensing(CS-) based approaches have been proposed [28-33].

Numerous preamble-based schemes have been proposed for CE. The interference approximation method (IAM) [20] and interference cancellation method (ICM) [21] are two well-known algorithms for interference mitigation. They reduce the imaginary interference or exploit this interference to improve CE performance. Combined with these methods, a novel preamble structure for FBMC systems was proposed in [22]. Because more channel coefficients need to be determined, CE in MIMO systems is more complicated than in single-input single-output (SISO) systems. In addition, imaginary interference from multiple antennas makes $\mathrm{CE}$ in MIMO-FBMC systems difficult. Thus, there has been significant research on CE for MIMO systems. In [23], IAM preamble variants were investigated and the characteristic due to the extension to MIMO systems was studied. An optimized preamble for a frequency division multiplexing MIMO system was proposed in [24]. In [25], an interference elimination MIMO preamble structure was proposed to improve CE performance. In addition, an efficient sequence design for a MIMO-FBMC system was proposed in [26]. However, the use of a preamble reduces spectrum efficiency, and it is difficult to remove the intrinsic interference. There is consensus [27] that preamble-based CE for MIMO systems is inefficient.

Many works had been devoted to improve the CE performance in FBMC systems. In [34], the authors proposed a blind CE method by utilizing spatial diversity to introduce data redundancy. However, the method could not provide satisfactory CE performance. By utilizing the sparse channel characteristics, the CS approach is explored to promote the CE performance. Most works [35-37] were reported on CE in OFDM systems. Only few studies can be found for FBMC systems. In [28], a traditional orthogonal matching pursuit (OMP) method was utilized for CE. The results obtained showed that compared with the traditional preamble structure scheme, this approach could significantly improve the CE performance. In [29], a sparse adaptive CS algorithm was put forward for high CE. This algorithm is based on the compressive sampling matching pursuit (CoSaMP) and sparsity adaptive matching pursuit (SAMP) methods. Besides, a scattered pilot CE method based on CS for FBMC was proposed in [30] by utilizing the wireless channel sparsity. In [31], the authors developed two distinctive compressive sensing algorithms to estimate channel frequency response in FBMC systems. Simulations verified the superiority of the two algorithms. In [32], an effective CS-based CE method was given for MIMO systems. A sparse adaptive scheme for $\mathrm{CE}$ in MIMO systems was proposed in [33]. However, no work has been reported on Bayesian compressive sensing (BCS) for CE in FBMC systems. As a special CS method, BCS, which utilizes statistical information of sparse channels as prior knowledge, can achieve better recovery effect than traditional CS methods in many applications [38, 39].

Motivated by those above, in this paper, we explore the statistical information of sparse channels for CE. The main contributions of this paper are listed as follows:
(1) Based on Bayesian CS approach, we propose an iterative fast Bayesian matching pursuit approach for high channel estimation in FBMC and its MIMO scenario. As far as we know, the Bayesian approach for high CE in FBMC systems has not yet been investigated

(2) To evaluate the performance of the proposed Bayesian approach, well-known CS methods and the least square (LS) method are utilized for comparison. Moreover, mean square error (MSE) and bit error rate (BER) are adopted to assess the CE performance. Simulations verify that the Bayesian approach can offer better both MSE and BER performance than other well-known CS approaches

The rest of this paper is organized as follows. Section 2 presents the system model, including FBMC and MIMOFBMC systems. Section 3 reviews the CS-based channel estimation method. In Section 4, a fast Bayesian matching pursuit channel estimation approach is proposed. In Section 5, the simulation comparisons are carried out and the results are analyzed. Finally, Section 6 gives the conclusions.

\section{System Model}

2.1. FBMC System. The FBMC signal can be expressed as

$$
s(t)=\sum_{m=0}^{N-1} \sum_{n} d_{m, n} g_{m, n}(t)
$$

where $N$ denotes the number of subcarriers, $g_{m, n}(t)$ represents the time-frequency prototype filter, and $d_{m, n}$ represents the real-valued OQAM symbol. The $(.)_{m, n}$, in which $m$ represents the subcarrier index and $n$ represents the symbol time index, denotes the $(m, n)$ th frequency-time (FT) point.

The filter functions $g_{m, n}$ are orthogonal in the real domain with

$$
\mathfrak{R}\left\{\left\langle g_{m, n} \mid g_{m_{0}, n_{0}}\right\rangle\right\}=\mathfrak{R}\left\{\sum_{t} g_{m, n}(t) g_{m_{0}, n_{0}}^{*}(t)\right\}=\delta_{m, m_{0}} \delta_{n, n_{0}}
$$

where $\mathfrak{R}(\cdot)$ represents the real part of a complex number and $\delta$ is the Kronecker delta function with $\delta_{m, m_{0}}=1$, if $m=m_{0}$ and $\delta_{m, m_{0}}=0$. Note that even without channel distortion, there is still imaginary intercarrier interference at the output of the filter bank. The weight of the interference is given by

$$
\langle g\rangle_{m, n}^{m_{0}, n_{0}}=-j\left\langle g_{m, n} \mid g_{m_{0}, n_{0}}\right\rangle
$$

where $\left\langle g_{m, n} \mid g_{m_{0}, n_{0}}\right\rangle$ denotes an imaginary term for $(m, n)$ $\neq\left(m_{0}, n_{0}\right)$. The values of interference weights can be 
calculated based on the prototype filter $g$, and thus, for all $m$, the interference weights are given by

$$
\begin{array}{ccc}
(-1)^{m} \varepsilon & 0 & -(-1)^{m} \varepsilon, \\
(-1)^{m} \delta & -\beta & (-1)^{m} \delta, \\
-(-1)^{m} \gamma & d_{m, n} & (-1)^{m} \gamma, \\
(-1)^{m} \delta & \beta & (-1)^{m} \delta, \\
(-1)^{m} \varepsilon & 0 & -(-1)^{m} \varepsilon .
\end{array}
$$

Generally, $\beta, \gamma>\delta$. In simulation, $\gamma=0.5004, \beta=0.3183$, $\delta=0.2501$, and $\varepsilon=0$ for the design of $g$.

Then, the received signal is given by

$$
r(t)=\sum_{k}^{K-1} s(t-k) h(t)+w(t)
$$

where $K$ is the number of channel taps, $w(t)$ is the additive white Gaussian noise (AWGN), and $h(t)$ is the time domain impulse response of the multipath channel. The channel can be described as

$$
h(t)=\sum_{k=0}^{K-1} a_{k}(t) \delta\left(\tau-\tau_{k}\right)
$$

where $a_{k}(t)$ denotes the complex amplitude of the $k$ th path, $\delta(\cdot)$ is the Kronecker delta, and $\tau_{k}$ represents the delay of the $k$ th path. It is assumed that it is a complex Gaussian process of wide-sensing stationary (WSS), and the channel paths are independent. Assuming that the length of channel impulse response is $L$, and the channel $\mathbf{h}=\left[h_{0}, h_{1}, \cdots h_{L-1}\right]^{T}$.

2.2. MIMO-FBMC System. For a $N_{t} \times N_{r}\left(N_{t} \leq N_{r}\right)$ spatial multiplexing MIMO system, the baseband signal on the $n_{t}$ th branch can be expressed as

$$
s^{n_{t}}(t)=\sum_{m=0}^{N-1} \sum_{n} d_{m, n}^{n_{t}} g_{m, n}(t)
$$

where $n_{t}=\left[1,2, \cdots, N_{t}\right]$ and $d_{m, n}^{n_{t}}$ is the real-valued FBMC/OQAM symbol on the $n_{t}$ th transmit antenna conveyed by subcarrier $m$ during symbol time $n$. $N$ is the number of subcarriers, and

$$
g_{m, n}(t)=g\left(t-n \tau_{0}\right) e^{i 2 \pi m F_{0} t} e^{j \phi_{m, n}}
$$

where $g(t)$ is a symmetric real-valued pulse filter, $F_{0}$ is the subcarrier spacing with $F_{0}=1 / T_{0}=1 / 2 \tau_{0}$, and $\phi_{m, n}$ is an additional phase term. $T_{0}$ is the OFDM symbol duration, and $\tau_{0}$ denotes the time offset between the real and imaginary parts of an FBMC/OQAM symbol.
The received signal can be expressed as

$$
r^{n_{r}}(t)=\sum_{n_{t}=1}^{N_{t}} \sum_{m=0}^{N-1} \sum_{n} H_{m, n}^{n_{r}, n_{t}}(t) d_{m, n}^{n_{t}} g_{m, n}(t)+\eta^{n_{r}}(t)
$$

with

$$
H_{m, n}^{n_{r}, n_{t}}(t)=\int_{0}^{\tau_{\max }} h_{m, n}^{n_{r}, n_{t}}(t, \tau) e^{-2 j \pi m F_{0} \tau} d \tau
$$

where $h_{m, n}^{n_{r}, n_{t}}(t, \tau)$ denotes the channel impulse response; $n_{r}$ and $n_{t}$ denote the receive and transmit antennas, respectively; $\eta^{n_{r}}(t)$ is the channel noise; and $H_{m, n}^{n_{r}, n_{t}}(t)$ is the complex channel response at time $t$. We assume a slowly varying channel, so we omit $t$ for brevity giving $H_{m, n}^{n_{r}, n_{t}}(t)=H_{m, n}^{n_{r}, n_{t}}$.

\section{CS-Based Channel Estimation}

Classical CS theory indicated that a $K$-spare signal $h$ could be stably reestablished as

$$
y=\Phi h+w
$$

with the precondition that $\Phi$ should meet the Restricted Isometry Property (RIP), $\Phi$ is a matrix with $M$ rows and $N$ columns, $M \ll N$, and $w$ is noise.

Equation (5) in matrix form can be expressed as

$$
R=X H+W
$$

with $R=[r(0), r(1), \cdots, r(N-1)]^{T}, X=\operatorname{diag}(x(0), x(1), \cdots$, $x(N-1)), W$ represents a $N \times N$ dimension noise matrix with zero mean and $\sigma^{2}$ variance, and $H=F_{N L} h$ is the channel frequency response, with $F_{N L}$ being an $L$-row discrete Fourier transform matrix, and $L$ denotes the channel length.

Let $P$ be the number of pilot signals and $\varphi$ the $P \times N$ pilot matrix with $\varphi=\left(e_{s_{1}}, e_{s_{2}}, \cdots, e_{s_{P}}\right)$. For the $N_{c}$ subcarriers, $\varphi$ is used to select the position of the pilot, and $s_{i}(i=1,2, \cdots, P)$ represents the position of the $i$ th pilot. Then, equation (12) can be written as follows:

$$
R_{P}=X_{P} F_{p} h+W_{P}
$$

where $R_{P}$ denotes the LS estimation channel value, $R_{P}=\varphi R$, and $W_{P}=\varphi W . X_{P}$ denotes a diagonal matrix, with $X_{P}=$ $\varphi X \varphi^{T}$, and the diagonal elements are pilot values, $F_{P}=\varphi F_{N L}$. Convert (13) to

$$
R_{P}=F h+W_{P}
$$

where $F=X_{P} F_{P}, R_{P}$ and $F$ are available during transmission, and $h$ represents the multipath channel impulse response. Then, the CS recovery algorithm can be used to recover $h$. 
Similarly, in MIMO systems, the CS method is also based on the above analysis. The received signal in (7) can be written as

$$
R^{n_{r}}=X^{n_{t}} H^{n_{r}, n_{t}}+\eta^{n_{r}}
$$

where $H^{n_{r}, n_{t}}$ is the channel frequency response given by $H^{n_{r}, n_{t}}=F_{N L} h^{n_{r}, n_{t}}, \quad X^{n_{t}}=\operatorname{diag}\left(x^{1}(0), x^{2}(1), \cdots, x^{n_{t}}(N-1)\right)$, $R^{n_{r}}=\left[r^{1}(0), r^{2}(1), \cdots, r^{n_{r}}(N-1)\right]^{T}, F_{N L}$ is the $N \times L$ discrete Fourier transform matrix, and $\eta^{n_{r}}$ is an $N \times N$ noise matrix with zero mean and variance $\sigma^{2}$.

Letting $P$ be the number of pilot signals, equation (15) can be rewritten as

$$
R_{P}^{n_{r}}=X_{P}^{n_{t}} F_{p} h^{n_{r}, n_{t}}+\eta_{P}
$$

where $R_{P}^{n_{r}}=\varphi R^{n_{r}}$ is the received pilot signals, $R_{P}^{n_{r}}$ is the LS estimated channel values, $\eta_{P}=\varphi \eta^{n_{r}}, X_{P}^{n_{t}}$ is a diagonal matrix with $X_{P}^{n_{t}}=\varphi X^{n_{t}} \varphi^{T}$, and the pilot values are $F_{P}=\varphi F_{N L}$. Equation (16) can be expressed as

$$
R_{P}^{n_{r}}=F h^{n_{r}, n_{t}}+\eta_{P}
$$

where $F=X_{P}^{n_{t}} F_{p}$ and $h^{n_{r}, n_{t}}$ is the channel impulse response. If $R_{P}^{n_{r}}$ and $F$ are available, then $h^{n_{r}, n_{t}}$ can be employed in the CS reconstruction algorithm.

Considering all receive antennas, equation (17) can be written as

$$
R=X h+\eta
$$

where $\quad X=I_{N_{r}} \otimes\left[\operatorname{diag}\left(x^{1}\right) F_{N L}, \operatorname{diag}\left(x^{2}\right) F_{N L}, \cdots, \operatorname{diag}\left(x^{N_{t}}\right)\right.$ $\left.F_{N L}\right], \quad R=\left[\left(R^{1}\right)^{T}\left(R^{2}\right)^{T} \cdots\left(R_{0}^{N_{r}}\right)^{T}\right]^{T}, \quad \eta=\left[\left(\eta^{1}\right)^{T} \quad\left(\eta^{2}\right)^{T} \ldots\right.$ $\left.\left(\eta^{N_{r}}\right)^{T}\right]^{T}$,

$$
h=\left[\begin{array}{cccc}
h^{1,1} & h^{1,2} & \cdots & h^{1, N_{t}} \\
h^{2,1} & h^{2,2} & \cdots & h^{2, N_{t}} \\
\vdots & \vdots & \ddots & \vdots \\
h^{N_{r}, 1} & h^{N_{r}, 2} & \cdots & h^{N_{r}, N_{t}}
\end{array}\right]
$$

\section{Proposed Bayesian Matching Pursuit Method}

It is considered that $\mathbf{h}$ is a Gaussian mixture process, the parametric vector $\mathbf{z}$ is introduced to reflect the sparsity of $\mathbf{h}$, and the position of nonzero elements is the same as that of h. $h[n]$ is defined as the $n$th element of vector $\mathbf{h}$, and $z[n]$ $\in\{0,1\}$ is used to express whether $h[n]$ is a nonzero element, since $h[n]$ is a Gaussian process with a mean of 0 and a variance of $\sigma^{2}$ :

$$
h[n] \mid\{z[n]=q\} \sim C N\left(0, \sigma_{q}^{2}\right)
$$

where $z[n]=0, \quad \sigma_{0}^{2} \triangleq 0, \quad h[n]=0, \quad z[n]=1, \quad h[n] \neq 0, \quad$ and $p(z[n]=1)=p_{1}$ and $p(z[n]=0)=1-p_{1}$.
The vector form of equation (20) can be expressed as follows:

$$
\mathbf{h} \mid \mathbf{z} \sim C N\left(0, \mathbf{R}_{\mathbf{z z}}\right),
$$

with $\mathbf{R}_{\mathbf{z z}}=E\left\{\mathbf{z z}^{\mathbf{H}}\right\}$.

Using Bayesian rule,

$$
p(\mathbf{y}, \mathbf{h} \mid \mathbf{z})=\frac{p(\mathbf{y}, \mathbf{h}, \mathbf{z})}{p(\mathbf{z})}=p(\mathbf{y} \mid \mathbf{z}, \mathbf{h}) p(\mathbf{h} \mid \mathbf{z})=p(\mathbf{y} \mid \mathbf{h}) p(\mathbf{h} \mid \mathbf{z}),
$$

if $\mathbf{h}$ is given, $\mathbf{z}$ can be completely determined, then $p(\mathbf{y} \mid \mathbf{z}, \mathbf{h})$ $=p(\mathbf{y} \mid \mathbf{h}, \mathbf{z})=p(\mathbf{y} \mid \mathbf{h})$.

$$
\left[\begin{array}{l}
\mathbf{y} \\
\mathbf{h}
\end{array}\right] \mid \mathbf{z}=\left[\begin{array}{c}
\mathbf{y} \mid \mathbf{z} \\
\mathbf{h} \mid \mathbf{z}
\end{array}\right] \sim C N\left(0,\left[\begin{array}{cc}
\varphi(\mathbf{z}) & \Phi \mathbf{R}_{\mathbf{z z}} \\
\mathbf{R}_{\mathbf{z z}} \Phi^{H} & \mathbf{R}_{\mathbf{z z}}
\end{array}\right]\right),
$$

where $\varphi(\mathbf{z}) \triangleq \Phi \mathbf{R}_{\mathbf{z z}} \Phi^{H}+\sigma_{n}^{2} \mathbf{I}_{\mathbf{M}}$.

According to the description of the above model, the selection of the support set for $\mathbf{h}$ can be simplified to the selection of the support set for $\mathbf{z}$. Using the Bayesian rule, the posterior probability could be written as

$$
p(\mathbf{z} \mid \mathbf{y})=\frac{p(\mathbf{y} \mid \mathbf{z}) p(\mathbf{z})}{\sum_{z^{\prime} \in \Lambda} p\left(\mathbf{y} \mid \mathbf{z}^{\prime}\right) p\left(\mid \mathbf{z}^{\prime}\right)},
$$

where $\Lambda \in\{0,1\}^{L} \cdot p(\mathbf{z} \mid \mathbf{y})$ can be obtained by solving $p(\mathbf{y} \mid \mathbf{z})$ $p(\mathbf{z})$; considering that $\Lambda$ has a relatively large value, it is still difficult to solve $p(\mathbf{y} \mid \mathbf{z}) p(\mathbf{z})$. If one can find a smaller set $\Lambda^{*}$ to approximate $\Lambda$, the amount of calculation could be reduced. In order to choose $\Lambda^{*}$, we first take the logarithm of $p(\mathbf{y} \mid \mathbf{z}) p(\mathbf{z})$,

$$
\begin{aligned}
v(\mathbf{z}) & \triangleq \ln p(\mathbf{y} \mid \mathbf{z}) p(\mathbf{z})=\ln p(\mathbf{y} \mid \mathbf{z})+\ln \prod_{n=1}^{L} p\left(z_{n}\right) \\
& =\ln p(\mathbf{y} \mid \mathbf{z})+\|\mathbf{z}\|_{0} \ln p_{1}+\left(N-\|\mathbf{z}\|_{0}\right) \ln \left(1-p_{1}\right),
\end{aligned}
$$

where $\ln p(\mathbf{y} \mid \mathbf{z})=-(L / 2) \ln 2 \pi-(1 / 2) \ln \operatorname{det}(\varphi(\mathbf{z}))-(1 / 2)$ $\mathbf{y}^{H} \varphi(\mathbf{z})^{-1} \mathbf{y}$ and $v(\mathbf{z})$ is the selection criterion for $\Lambda^{*}$. Then, an effective way is taken to estimate $\mathbf{z}$ by utilizing $v(\mathbf{z})$, and we call this method as the fast Bayesian matching pursuit (FBMP) [32].

More specifically, suppose $\mathbf{z}_{\mathbf{n}}$ means that the vector is the same as the vector $\mathbf{z}$ except for the $n$th element, with $z_{n}[n]=1, \mathrm{z}[n]=0$. Then, calculate the gain of $v\left(\mathbf{z}_{n}\right)$, with $\Delta_{n}\left(\mathbf{z}_{n}\right)=v\left(\mathbf{z}_{n}\right)-v(\mathbf{z})$. According to (23), $\varphi(0)=\sigma_{n}^{2} I_{M}$. Notice the initialization state of $\mathbf{z}$; when $\mathbf{z}=0, v(0)=-(L / 2)$ $\ln 2 \pi-M \ln \sigma_{n}-\left(1 / 2 \sigma_{n}^{2}\right)\|y\|_{2}^{2}+L \ln \left(1-p_{1}\right)$. In order to get the gain of $v\left(\mathbf{z}_{n}\right)$, we first calculate

$$
\begin{aligned}
\varphi\left(\mathbf{z}_{n}\right) & =\Phi \mathbf{R}_{\mathbf{z} n \mathbf{z} n} \Phi^{H}+\sigma_{n}^{2} \mathbf{I}_{\mathbf{M}}=\Phi\left(\mathbf{R}_{\mathbf{z z}}+\mathbf{A}\right) \Phi^{H}+\sigma_{n}^{2} \mathbf{I}_{\mathbf{M}} \\
& =\varphi(\mathbf{z})+\sigma_{1}^{2} \Phi_{n} \Phi_{n}^{H},
\end{aligned}
$$


where $\mathbf{A}$ is an $L \times L$ matrix; it has zero elements except for $A[n, n]$, and $A[n, n]=\sigma_{z n}^{2}=\sigma_{1}^{2} . \Phi_{n}$ represents the $n$th column of matrix $\Phi$. Then, according to the principle of matrix transpose

$\varphi\left(\mathbf{z}_{n}\right)^{-1}=\varphi(\mathbf{z})^{-1}-\varphi(\mathbf{z})^{-1} \Phi_{n}\left(\Phi_{n}^{H} \varphi(\mathbf{z})^{-1} \Phi_{n}+\sigma_{1}^{-2}\right)^{-1} \Phi_{n}^{H} \varphi(\mathbf{z})^{-1}$

Define that $\mathbf{b}_{n} \triangleq \varphi(\mathbf{z})^{-1} \Phi_{n}$ and $\beta_{n} \triangleq \sigma_{1}^{2}\left(1+\sigma_{1}^{2} \Phi_{n}^{H} \mathbf{b}_{n}\right)^{-1}$. Then, equation (27) can be rewritten as

$$
\varphi\left(\mathbf{z}_{n}\right)^{-1}=\varphi(\mathbf{z})^{-1}-\beta_{n} \mathbf{b}_{n} \mathbf{b}_{n}^{H} .
$$

Through calculation, we can finally get that

$\Delta_{n}\left(\mathbf{z}_{n}\right)=v\left(\mathbf{z}_{n}\right)-v(\mathbf{z})=\frac{1}{2} \ln \left(\frac{\beta_{n}}{\sigma_{1}^{2}}\right)+\frac{1}{2} \beta_{n}\left|\mathbf{y}^{H} \mathbf{b}_{n}\right|^{2}+\ln \frac{p_{1}}{1-p_{1}}$,

where $\Delta_{n}\left(\mathbf{z}_{n}\right)$ is the gain of $\mathbf{z}$ after changing at the $n$th position.

According to the above method, the main support set $\Lambda^{*}$ can be found. The steps of the algorithm are shown below:

Initialization: $\Omega=\varnothing, \mathbf{z}=0, \mathbf{R}_{\mathbf{z z}}=0$, and $v(0)$. Use (23) to obtain $\varphi(\mathbf{z})$, and use (26) to initialize $\mathbf{b}_{n}, n=1,2, \cdots, L$, and $\beta_{n}$.

First, starting with $\mathbf{z}=0$, use (29) to calculate $\Delta_{n}\left(\mathbf{z}_{n}\right)$, $n=1: L$, find the location element with the largest $v\left(\mathbf{z}_{n}\right)=v(0)+\Delta_{n}\left(\mathbf{z}_{n}\right), n=1: L$, and record it as $n^{(1)}$; add it to the set $\Omega=\Omega \cup\left\{n^{(1)}\right\}$, then update $\Psi=\{1,2, \cdots, L\} / \Omega$.

Then, update $\mathbf{b}_{n}$ and $\beta_{n}$, use (29) to calculate $\Delta_{n}\left(\mathbf{z}_{n}\right)$, $n=1: L$, find the location element with the largest $v\left(\mathbf{z}_{n}\right)=v(\mathbf{z})+\Delta_{n}\left(\mathbf{z}_{n}\right), n=1: L$, and record it as $n^{(2)}$.

Until the number of elements in the selection $\Omega$ reaches $\widehat{K}$, set $\widehat{K}$ to be slightly greater than the expected position of nonzero elements, with $E\left\{\|\mathbf{z}\|_{0}\right\}=L p_{1}$. Therefore, the probability that the actual sparsity is greater than the estimated sparsity $\widehat{K}$ is lower, and $P_{0}=p\left(\|\mathbf{z}\|_{0}>\widehat{K}\right)=(1 / 2)$ erfc $\left(\left(\widehat{K}-L p_{1}\right) / \sqrt{2 N p_{1}\left(1-p_{1}\right)}\right) ; \quad \widehat{K} \quad$ could be calculated as $\widehat{K}=\left[\operatorname{erfc}^{-1}\left(2 p_{0}\right) \sqrt{2 L p_{1}\left(1-p_{1}\right)}+L p_{1}\right]$.

In the proposed algorithm, by giving the value of $P_{0}$, use the above formula to calculate $\widehat{K}$, until the number of elements in $\Omega$ reaches $\widehat{K}$. By optimizing the number of loops, the iteration will be stopped until the requirements are met.

After estimating the parameter vector $\mathbf{z}$, the estimated channel is given as

$$
\widehat{\mathbf{h}}=\sum_{\Lambda^{*}} E\{\mathbf{h} \mid \mathbf{y}, \mathbf{z}\} p(\mathbf{z} \mid \mathbf{y}),
$$

where $\{\mathbf{h} \mid \mathbf{y}, \mathbf{z}\}=\mathbf{R}_{\mathbf{z z}} \Phi^{H} \Phi\left(\mathbf{z}_{n}\right)^{-1} \mathbf{y}$. Figure 1 shows the flowchart of the proposed algorithm.

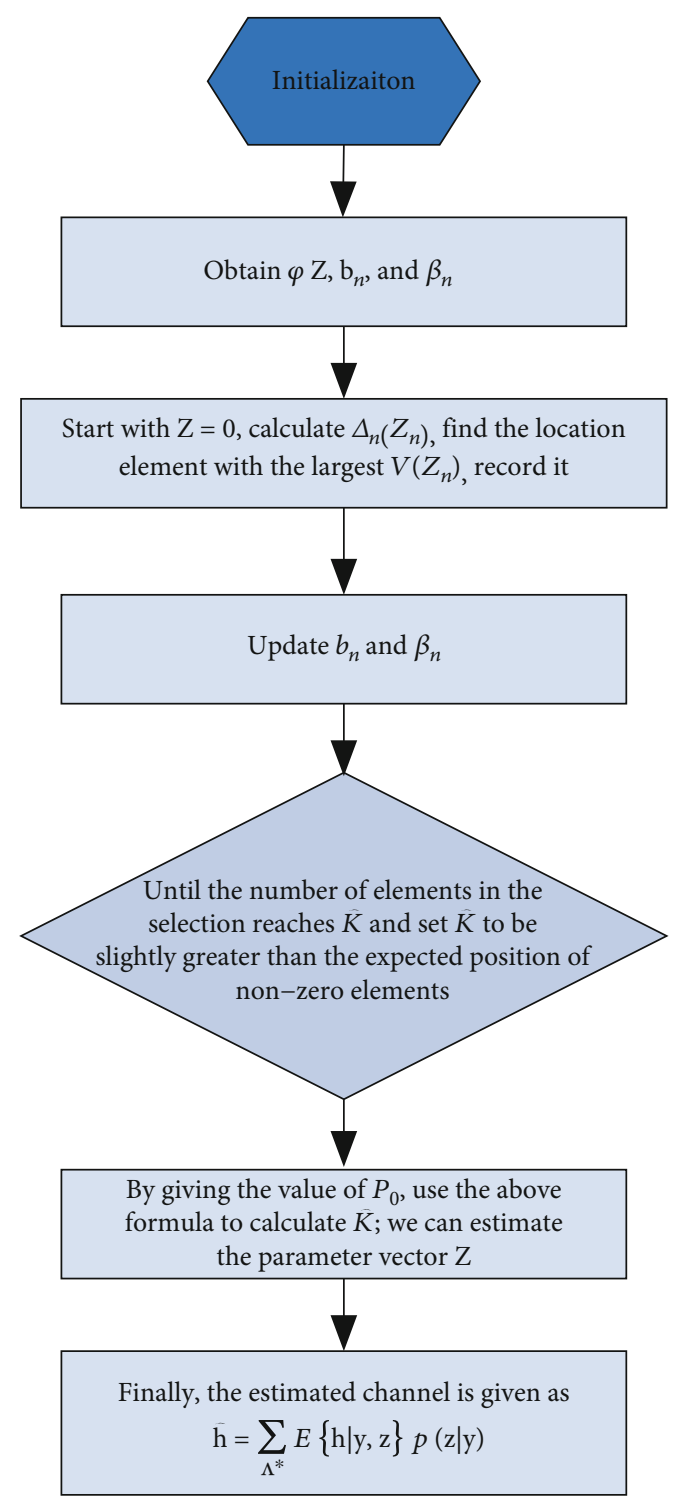

FIgURE 1: The flowchart of the proposed algorithm.

\section{Simulation Results}

In this section, we consider FBMC systems using $N=512$ subcarriers. A square root-raised cosine filter is adopted for the pulse filter. The LS approach adopts the IAM preamble structure. The length of the channel is $L=240$, the number of nonzero elements $K=6, K$ is also expressed as sparsity, and set $P_{0}=0.01$. It is clearly that $p_{1}=0.025$. The number of iterations is 5. MSE and BER are used to evaluate the CE performance. Conventional LS, OMP, and regularized OMP (ROMP) methods are used to compare with the proposed method, where the OMP and ROMP are the two wellknown CS recovery algorithms.

Figures 2 and 3 give the MSE and BER with five methods for a SISO-FBMC system. These results show that the proposed scheme outperforms the other four schemes in terms of both the MSE and BER. The CS-based approaches provide significant MSE performance improvement over the 


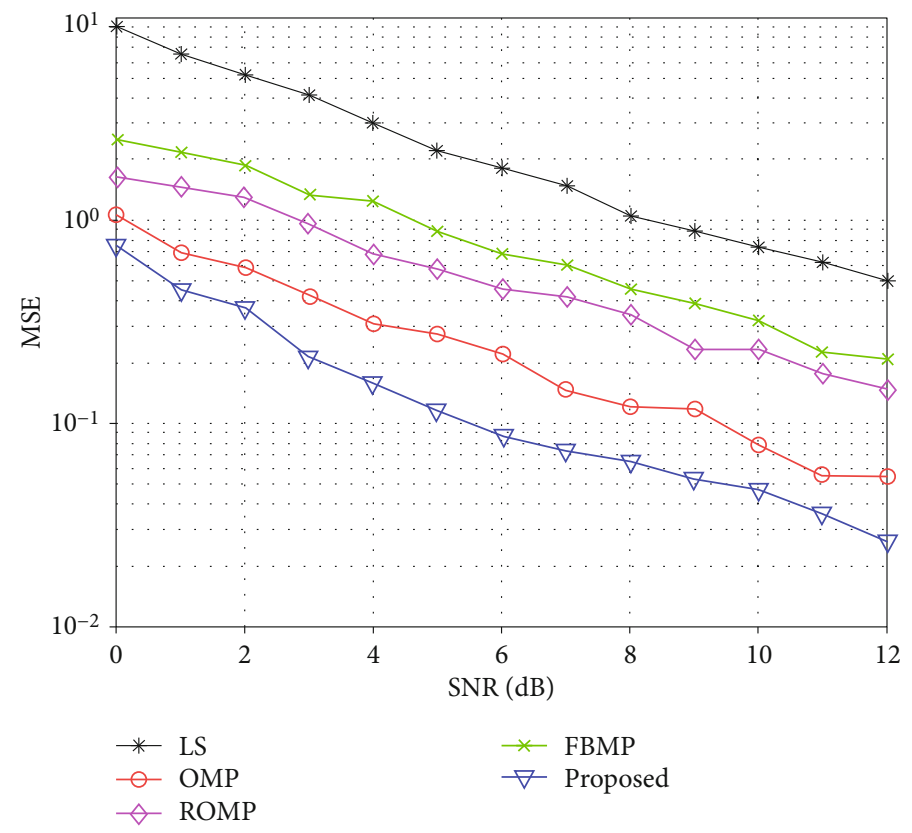

FIgURE 2: MSE with five methods for a SISO system.

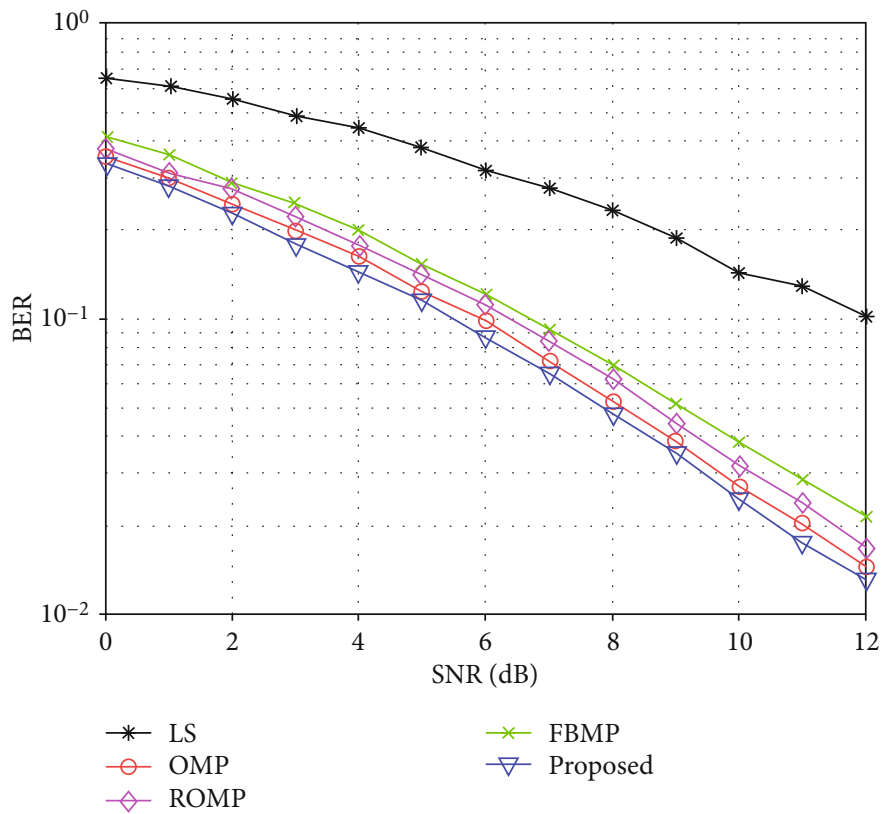

FIGURE 3: BER with five methods for a SISO system.

conventional LS method. Due to the change of iteration conditions, the proposed method is significantly better than the conventional FBMP method.

Figure 2 depicts the MSE performance curves for five channel estimation methods. The results show that our proposed algorithm exhibits a better MSE performance than the other four methods. The conventional FBMP method outperforms the LS method but is worse than the traditional
CS method. The MSE performance for CE can be significantly improved by the proposed method.

In Figure 3, the BER performance curves for five channel estimation methods are given. It is obvious that the proposed method gives the best BER values among the five methods. More specifically, our proposed approach improves the BER performance of the OMP, ROMP, and FBMP by 0.5 , 0.8 , and $1.2 \mathrm{~dB}$, respectively, when $B E R=10^{-1}$ is considered. 


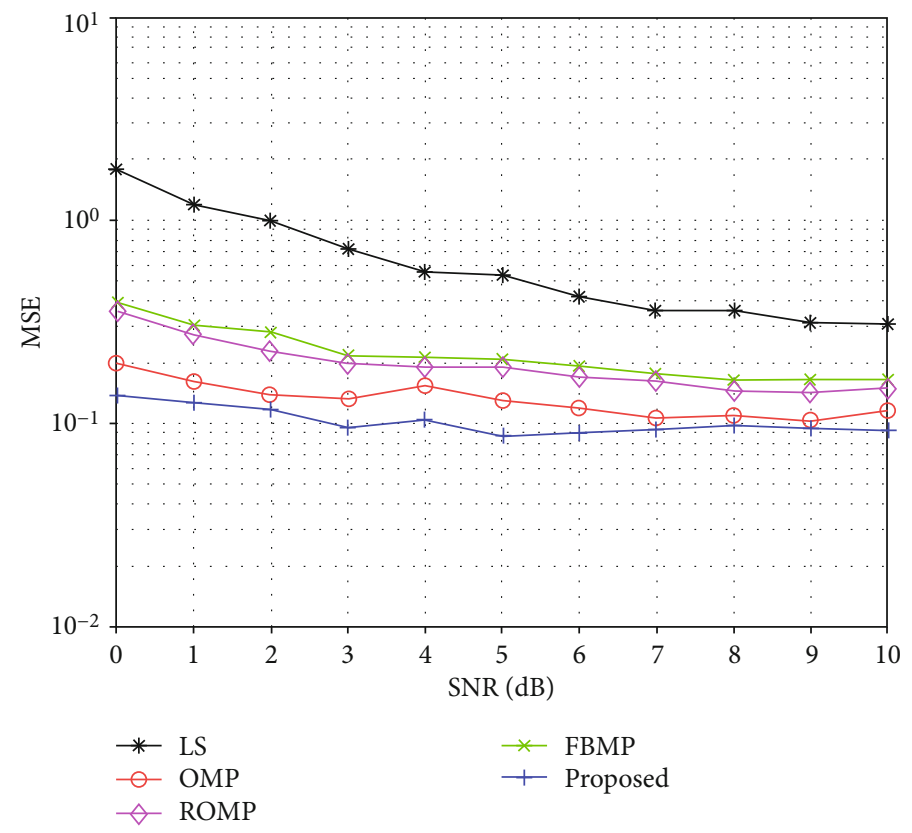

Figure 4: MSE with five methods for a $2 \times 2$ MIMO system.

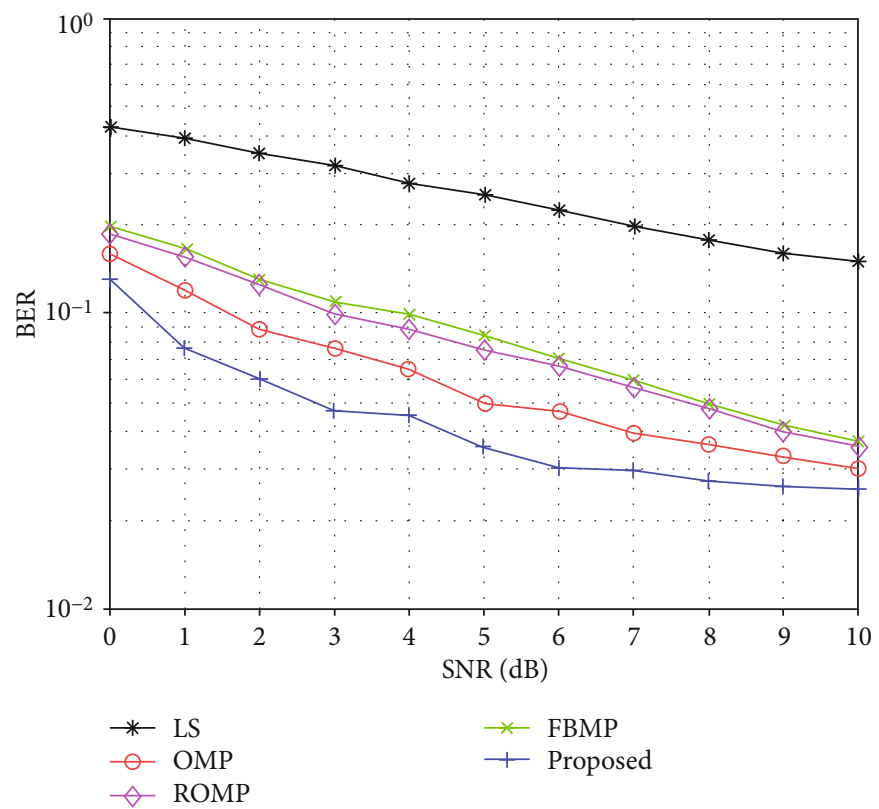

FIGURE 5: BER with five methods for a $2 \times 2$ MIMO system.

We also study the CE performance of a MIMO-FBMC system. Figures 4 and 5 give the MSE and BER of the five methods for a $2 \times 2$ MIMO-FBMC system. Compared with the SISO system, the performance of all methods in the MIMO system is decreased. And these results also demonstrate that the proposed method outperforms the other four schemes in terms of both the MSE and BER in a MIMO case.

Figure 4 gives the MSE performance curves for five channel estimation methods in a MIMO case. Compared with the performance in Figure 2, the OMP method offers slightly better MSE values than the FBMP method in Figure 4. The error floor of the CS-based approaches is due to the inherent interference from the MIMO-FBMC system.

Figure 5 shows that the CS approach outperforms the conventional LS scheme in terms of the BER, and the proposed method provides the best performance. More specifically, the proposed approach improves the BER performance of the OMP, ROMP, and FBMP by 1.1, 
2.6, and 3.6 dB, respectively, when $\mathrm{BER}=10^{-1}$ is considered. The BER performance improvement of the proposed method in a MIMO system is better than that in a SISO system.

\section{Conclusions}

In this paper, we had studied the FBMP algorithm for channel estimation. A modified FBMP algorithm was proposed by optimizing the iterative termination conditions for FBMC sparse channel estimation. The proposed algorithm was compared with the LS, OMP, ROMP, and FBMP methods. The simulation results obtained showed that our proposed approach achieved better MSE and BER performance than LS and the other well-known CS-based approaches. However, the shortcoming is that the computational complexity of the proposed method is increased. In the future, lowcomplexity Bayesian sparse CE approach for FBMC systems will be studied.

\section{Data Availability}

The data used to support the findings of this study are available from the corresponding author upon reasonable request and with permission from the funders.

\section{Conflicts of Interest}

The authors declare that there is no conflict of interests regarding the publication of this paper.

\section{Acknowledgments}

This work was supported by the National Natural Science Foundation of China (Grant Nos. 61901409, 61861015, 61961013, and 61661030), Opening Foundation of Key Laboratory of Opto-technology and Intelligent Control (Lanzhou Jiaotong University), Ministry of Education (Grant No. KFKT2019-2), Shandong Province Natural Science Foundation (No. ZR2017BF023), Shandong Province Postdoctoral Innovation Project (No. 201703032), Doctoral Found of QUST (Grant No. 010029029), Key Research and Development Program of Hainan Province (No. 311 ZDYF2019011), Young Elite Scientists Sponsorship Program by CAST (No. 2018QNRC001), and Macau Foundation (No. MF1909).

\section{References}

[1] L. Xu, J. Wang, H. Wang, T. Aaron Gulliver, and K. N. le, "BP neural network-based ABEP performance prediction for mobile Internet of Things communication systems," Neural Computing and Applications, 2019.

[2] L. Chettri and R. Bera, "A comprehensive survey on Internet of Things (IoT) toward 5G wireless systems," IEEE Internet of Things Journal, vol. 7, no. 1, pp. 16-32, 2020.

[3] F. Wen, J. Shi, and Z. Zhang, "Joint 2D-DOD, 2D-DOA, and polarization angles estimation for bistatic EMVS-MIMO radar via PARAFAC analysis," IEEE Transactions on Vehicular Technology, vol. 69, no. 2, pp. 1626-1638, 2020.
[4] F. Wen, J. Wang, J. Shi, and G. Gui, "Auxiliary vehicle positioning based on robust DOA estimation with unknown mutual coupling," IEEE Internet of Things Journal, 2020.

[5] L. Lyu, C. Chen, S. Zhu, and X. Guan, "5G enabled codesign of energy-efficient transmission and estimation for industrial IoT systems," IEEE Transactions on Industrial Informatics, vol. 14, no. 6, pp. 2690-2704, 2018.

[6] X. Li, J. Li, and L. Li, "Performance analysis of impaired SWIPT NOMA relaying networks over imperfect Weibull channels," IEEE Systems Journal, vol. 14, no. 1, pp. 669-672, 2020.

[7] X. Li, M. Liu, C. Deng, P. T. Mathiopoulos, Z. Ding, and Y. Liu, "Full-duplex cooperative NOMA relaying systems with I/Q imbalance and imperfect SIC," IEEE Wireless Communications Letters, vol. 9, no. 1, pp. 17-20, 2020.

[8] X. Li, J. Li, Y. Liu, Z. Ding, and A. Nallanathan, "Residual transceiver hardware impairments on cooperative NOMA networks," IEEE Transactions on Wireless Communications, vol. 19, no. 1, pp. 680-695, 2020.

[9] L. Xu, J. Wang, Y. Liu, W. Shi, and T. A. Gulliver, "Outage performance for IDF relaying mobile cooperative networks," Mobile Networks and Applications, vol. 23, no. 6, pp. 14961501, 2018.

[10] X. Wang, L. Wan, M. Huang, C. Shen, and K. Zhang, "Polarization channel estimation for circular and non-circular signals in massive MIMO systems," IEEE Journal of Selected Topics in Signal Processing, vol. 13, no. 5, pp. 1001-1016, 2019.

[11] H. Wang, L. Wan, M. Dong, K. Ota, and X. Wang, “Assistant vehicle localization based on three collaborative base stations via SBL-based robust DOA estimation," IEEE Internet of Things Journal, vol. 6, no. 3, pp. 5766-5777, 2019.

[12] X. Wang, L. Wan, M. Huang, C. Shen, Z. Han, and T. Zhu, "Low-complexity channel estimation for circular and noncircular signals in virtual MIMO vehicle communication systems," IEEE Transactions on Vehicular Technology, vol. 69, no. 4, pp. 3916-3928, 2020.

[13] K. Zheng, Z. Yang, K. Zhang, P. Chatzimisios, K. Yang, and W. Xiang, "Big data-driven optimization for mobile networks toward 5G," IEEE Network, vol. 30, no. 1, pp. 44-51, 2016.

[14] X. Zhang and Q. Zhu, "Information-centric virtualization for software-defined statistical QoS provisioning over 5G multimedia big data wireless networks," IEEE Journal on Selected Areas in Communications, vol. 37, no. 8, pp. 1721-1738, 2019.

[15] R. Nissel and M. Rupp, "OFDM and FBMC-OQAM in doubly-selective channels: calculating the bit error probability," IEEE Communications Letters, vol. 21, no. 6, pp. 1297$1300,2017$.

[16] L. Zhang, P. Xiao, A. Zafar, A. Quddus, and R. Tafazolli, "FBMC system: an insight into doubly dispersive channel impact," IEEE Transactions on Vehicular Technology, vol. 66, no. 5, pp. 3942-3956, 2016.

[17] R. Nissel, S. Schwarz, and M. Rupp, "Filter bank multicarrier modulation schemes for future mobile communications," IEEE Journal on Selected Areas in Communications, vol. 35, no. 8, pp. 1768-1782, 2017.

[18] D. Chen, Y. Tian, D. Qu, and T. Jiang, "OQAM-OFDM for wireless communications in future internet of things: a survey on key technologies and challenges," IEEE Internet of Things Journal, vol. 5, no. 5, pp. 3788-3809, 2018.

[19] D. Gregoratti and X. Mestre, "Uplink FBMC/OQAM-based multiple access channel: distortion analysis under strong 
frequency selectivity," IEEE Transactions on Signal Processing, vol. 64, no. 16, pp. 4260-4272, 2016.

[20] J. F. Du and S. Signeel, "Novel preamble-based channel estimation for OFDM/OQAM systems," in Proceedings 2009 IEEE International Conference on Communications, pp. 1-6, Dresden, Germany, June 2009.

[21] E. Kofidis and D. Katselis, "Improved interference approximation method for preamble-based channel estimation in FBMC/OQAM," in Proceedings IEEE European Signal Process. Conference, pp. 1603-1607, Barcelona, Spain, September 2011.

[22] H. Wang, W. C. Du, and L. W. Xu, "Novel preamble design for channel estimation in FBMC/OQAM systems," KSII Transactions on Internet and Information Systems, vol. 10, no. 8, pp. 3672-3688, 2016.

[23] E. Kofidis, "Preamble-based estimation of highly frequency selective channels in FBMC/OQAM systems," IEEE Transactions on Signal Processing, vol. 65, no. 7, pp. 1855-1868, 2017.

[24] W. Liu, D. Chen, K. Luo, T. Jiang, and D. Qu, "FDMstructured preamble optimization for channel estimation in MIMO-OQAM/FBMC systems," IEEE Transactions on Wireless Communications, vol. 17, no. 12, pp. 8433-8443, 2018.

[25] H. Wang, L. Xu, X. Wang, and S. Taheri, "Preamble design with interference cancellation for channel estimation in MIMO-FBMC/OQAM systems," IEEE Access, vol. 6, pp. 44072-44081, 2018.

[26] J. Zhang, S. Hu, Z. Liu, P. Wang, P. Xiao, and Y. Gao, "Realvalued orthogonal sequences for iterative channel estimation in MIMO-FBMC systems," IEEE Access, vol. 7, pp. 6874268751, 2019.

[27] Z. L. Liu, P. Xiao, and S. Hu, "Low-PAPR preamble design for FBMC systems," IEEE Transactions on Vehicular Technology, vol. 68, no. 8, pp. 7869-7876, 2019.

[28] X. M. Liu, Z. W. Cai, A. L. Jia, and J. L. Ou, “A novel channel estimation method based on compressive sensing for OFDM/OQAM systems," The Journal of Computer Information Systems, vol. 9, no. 15, pp. 5955-5963, 2013.

[29] H. Wang, W. du, and L. Xu, "A new sparse adaptive channel estimation method based on compressive sensing for FBMC/OQAM transmission network," Sensors, vol. 16, no. 7, p. 966, 2016.

[30] L. Xiaopeng, C. Xihong, X. Zedong, Z. Kai, and T. Ningning, "Scattered pilots aided channel estimation based on compressed sensing in OQAM/OFDM system," Journal of National University of Defense Technology, vol. 39, no. 5, pp. 102-107, 2017.

[31] Z. He, L. Zhou, Y. Yang, Y. Chen, X. Ling, and C. Liu, "Compressive sensing-based channel estimation for FBMC-OQAM system under doubly selective channels," IEEE Access, vol. 7, pp. 51150-51158, 2019.

[32] M. Lin, Y. Li, L. Xiao, and J. Wang, "A compressive sensing channel estimation for MIMO FBMC/OQAM system," Wireless Personal Communications, vol. 96, no. 3, pp. 3345-3360, 2017.

[33] H. Wang, "Sparse channel estimation for MIMO-FBMC/OQAM wireless communications in smart city applications," IEEE Access, vol. 6, pp. 60666-60672, 2018.

[34] H. Wang, J. Liao, L. Xu, and X. Wang, "Blind channel estimation for FBMC/OQAM systems based on subspace approach," Information, vol. 9, no. 3, p. 58, 2018.
[35] J. Meng, W. Yin, Y. Li, N. T. Nguyen, and Z. Han, “Compressive sensing based high-resolution channel estimation for OFDM system," IEEE Journal of Selected Topics in Signal Processing, vol. 6, no. 1, pp. 15-25, 2012.

[36] C. Qi and L. Wu, "Uplink channel estimation for massive MIMO systems exploring joint channel sparsity," Electronics Letters, vol. 50, no. 23, pp. 1770-1772, 2014.

[37] R. Mohammadian, A. Amini, and B. H. Khalaj, "Compressive sensing-based pilot design for sparse channel estimation in OFDM systems," IEEE Communications Letters, vol. 21, no. 1, pp. 4-7, 2017.

[38] Z. Sadeghigol, M. H. Kahaei, and F. Haddadi, "Generalized beta Bayesian compressive sensing model for signal reconstruction," Digital Signal Processing, vol. 60, no. 1, pp. 163171, 2017.

[39] S. Liu, S. Wu, and Y. Li, "Application of Bayesian compressive sensing in IRUWB channel estimation," China Communications, vol. 14, no. 5, pp. 30-37, 2017. 